Linguistique, littérature, didactique

163-164 | 2014

Questions de morale. Éducation, discours, texte

\title{
Instruire et éduquer sur fond d'éthique
}

English title: Teaching and educating on an ethical background

\section{Claudine Leleux}

\section{(2) OpenEdition \\ Journals}

Édition électronique

URL : http://journals.openedition.org/pratiques/2237

DOI : $10.4000 /$ pratiques.2237

ISSN : 2425-2042

\section{Éditeur}

Centre de recherche sur les médiations (CREM)

\section{Édition imprimée}

Date de publication : 31 décembre 2014

\section{Référence électronique}

Claudine Leleux, «Instruire et éduquer sur fond d'éthique », Pratiques [En ligne], 163-164 | 2014, mis en ligne le 31 décembre 2014, consulté le 30 avril 2019. URL : http://journals.openedition.org/ pratiques/2237 ; DOI : 10.4000/pratiques.2237

Ce document a été généré automatiquement le 30 avril 2019.

(c) Tous droits réservés 


\title{
Instruire et éduquer sur fond d'éthique
}

\author{
English title: Teaching and educating on an ethical background
}

\author{
Claudine Leleux
}

1 Enseigner la morale laïque, comme on le dirait dans l'Hexagone, la morale non confessionnelle, comme on le pratique en Belgique, ou éduquer à la citoyenneté, exige comme tout enseignement de remplir trois conditions : l'enseignant doit faire preuve d'un savoir-faire professionnel, pouvoir s'appuyer sur une didactique spécifique et respecter une déontologie propre à cette discipline.

2 Si comme nous le verrons plus loin, la morale, l'éthique, voire la citoyenneté, sont à l'œuvre dans tout savoir ou dans toute vie de classe, alors les enseignants qui n'ont pas été formés à cette discipline doivent cependant pouvoir répondre de cette triple exigence.

3 La présente contribution, après avoir clarifié quelques concepts fréquemment utilisés dans la discipline d'éducation à la moralité, ciblera deux objectifs de la didactique spécifique (développer le jugement normatif et évaluatif) et de la déontologie spécifique (respecter le pluralisme éthique et éduquer sans moraliser, c'est-à-dire éduquer à l'autonomie du jugement).

\section{Présupposition pragmatique de l'entente}

4 Avant d'entrer dans le vif du sujet, il importe de clarifier quelques concepts-clés qui seront ici utilisés, avec, si nécessaire, leur auteur de référence parce qu'en matière d'éthique, de citoyenneté, de valeurs et de normes, nous pouvons à peu près lire tout et son contraire.

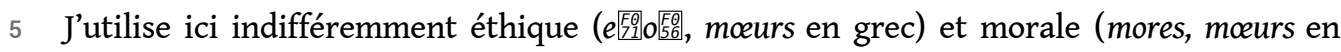
latin) sauf quand le contenu philosophique diffère selon l'un ou l'autre terme, par exemple lorsqu'il sera question plus tard de normes éthiques au sens téléologique aristotélicien ou de normes morales au sens de l'impératif catégorique de Kant. « Morale 
laïque » (en France), moralité publique, citoyenneté démocratique et Sittlichkeit au sens d'Hegel sont considérés ici comme quasi-synonymes.

6 J. Habermas a montré, notamment pour surmonter une difficulté en philosophie et en logique, que nous étions obligés de présupposer une prétention à l'entente dans tout agir et en particulier dans tout agir argumentatif ${ }^{1}$. Si nous définissons très succinctement l'éthique comme l'« ensemble des principes moraux qui sont à la base de la conduite de quelqu'un» (Larousse), la présupposition nécessaire d'un agir pour s'entendre met l'éthique à la base de tout savoir. Tout savoir est en effet un accord sur une prétention à l'exactitude ou la justesse des énoncés que nous proférons sur le monde. Cela ne veut pas dire que tout savoir est exact ou juste, mais qu'on se sera mis d'accord entre êtres humains pour accepter de le considérer comme exact ou juste.

7 D'un point de vue épistémologique, le savoir peut en gros se diviser en trois types de prétentions à la validité. J. Habermas considère que l'ensemble des énoncés descriptifs et constatifs prétendent à l'exactitude et constituent le savoir scientifique. En revanche, les savoirs moral et juridique rassemblent les énoncés normatifs et prescriptifs qui prétendent à ce que J. Habermas appelle la justesse normative (prétention d'une norme à être juste). Enfin, et cela reste aujourd'hui le savoir le plus problématique parce que le plus subjectif, l'ensemble des énoncés évaluatifs et expressifs qui prétendent à l'authenticité, c'est-à-dire à l'accord entre un sujet et ce qu'il connait et dit de lui-même.

Dans cette architectonique, nous retrouvons la partition kantienne des trois Critiques mais dans une perspective " postmétaphysique » cette fois. Chez J. Habermas, le passage d'une prétention à l'exactitude à l'exactitude ou d'une prétention à la justesse normative à la justesse normative est en effet provisoire et toujours à postériori. Car il est issu d'une discussion effective avec toutes les personnes concernées ${ }^{2}$ : la discussion permettra de vérifier si la prétention peut être honorée, si ce qui prétend être exact est exact ou ce qui prétend être juste est juste.

9 Nous pourrions schématiser cette architectonique ainsi dans le tableau1, en nous inspirant non seulement de J. Habermas mais aussi de J.-M. Ferry (1991).

Tableau 1. Architectonique de la raison et des savoirs.

\begin{tabular}{|c|c|c|c|}
\hline \multicolumn{4}{|l|}{ Intelligibilité } \\
\hline Énoncés & $\begin{array}{l}\text { constatifs } \\
\text { descriptifs }\end{array}$ & normatifs et prescriptifs & évaluatifs et expressifs \\
\hline $\begin{array}{l}\text { Prétention à } \\
\text { la validité }\end{array}$ & $\begin{array}{l}\text { l'exactitude } \\
\text { (le « vrai ») }\end{array}$ & $\begin{array}{l}\text { la justesse normative } \\
\text { (le «bien», le «juste», le } \\
\text { « légitime ») }\end{array}$ & $\begin{array}{l}\text { l'authenticité (le «bon», } \\
\text { la « valeur ») }\end{array}$ \\
\hline $\begin{array}{l}\text { Rapport au } \\
\text { monde }^{3}\end{array}$ & physique & Intersubjectif & subjectif \\
\hline $\begin{array}{l}\text { Sphère } \quad \mathrm{du} \\
\text { savoir }\end{array}$ & $\begin{array}{l}\text { science } \quad \& \\
\text { technique }\end{array}$ & droit \& morale & esthétique \\
\hline Catégorie & $\begin{array}{l}\text { faits } \\
\text { (ce qui est) }\end{array}$ & $\begin{array}{l}\text { normes } \\
\text { (ce qui doit être) }\end{array}$ & $\begin{array}{l}\text { valeurs } \\
\text { (le bien-être) }\end{array}$ \\
\hline
\end{tabular}




\begin{tabular}{|c|c|c|c|}
\hline Mode & indicatif & impératif & subjonctif \\
\hline $\begin{array}{l}\text { Personne } \\
\text { pronominale }\end{array}$ & il & tu & $\mathrm{je}$ \\
\hline $\begin{array}{l}\text { Validité de } \\
\text { l'énoncé }\end{array}$ & $\begin{array}{l}\text { à l'issue d'une } \\
\text { discussion } \\
\text { "sérieuse » par la } \\
\text { communauté des } \\
\text { savants }\end{array}$ & $\begin{array}{l}\text { à l'issue d'une discussion } \\
\text { «sérieuse » avec le public des } \\
\text { personnes concernées par la } \\
\text { norme (selon qu'elle soit } \\
\text { technique, éthique, morale ou } \\
\text { juridique) }\end{array}$ & $\begin{array}{l}\text { (après mûre réflexion, } \\
\text { clarification de l'accord } \\
\text { avec soi-même, voire } \\
\text { investigation de type } \\
\text { thérapeutique) }\end{array}$ \\
\hline
\end{tabular}

10 Après avoir rappelé la position habermassienne d'une transcendance de l'éthique (la nécessité de présupposer pragmatiquement une entente à tout agir), l'intérêt de l'architectonique ci-dessus est aussi :

1. de différencier deux niveaux de réflexion: d'une part, la «matière » d'une éducation morale et citoyenne et, d'autre part, le fond éthique sur lequel se meut toute éducation et toute instruction;

2. de distinguer une éducation aux valeurs et une éducation aux normes ;

3. de mettre en exergue à la fois l'intérêt de la grammaire dans l'éducation scolaire pour distinguer les faits, les normes et les valeurs : ce qui est, ce qui doit être ou ce qui est de l'ordre du bien-être ;

4. de différencier deux types de temps, le temps physique et le temps de l'idéal normatif, l'indicatif et le subjonctif, et, du coup, de relever non seulement l'intérêt de la conjugaison mais du recours à la narration comme ce pont, dit J. Bruner (2010:16), « entre ce qui est établi et ce qui est possible »;

5. de faire ressortir la nécessité de l'apprentissage de la discussion pour valider ou invalider les savoirs.

11 Cette architectonique sert dès lors de cadre de référence dans la suite du texte.

\section{Éduquer moralement?}

12 Une forte tradition française vise, depuis la Révolution de 1789 et les Mémoires sur l'instruction publique de N. de Condorcet (1791-1792), à réserver à la famille le soin d'éduquer les jeunes et de limiter la mission de l'école à l'instruction. Pourquoi ? Parce que l'école risquerait d'empiéter sur le droit des familles à éduquer leurs enfants comme ils l'entendent, c'est-à-dire selon leurs convictions éthiques ${ }^{4}$. Nous verrons que ce risque existe si l'enseignant ne se conforme pas à une déontologie spécifique en la matière.

Instruire voudrait dire aujourd'hui transmettre un savoir (au sens de science) établi par la communauté des savants. L'école n'étant pas le lieu d'élaboration du savoir, l'enseignant aurait la tâche (pédagogique) de faire reconstruire par les élèves les raisons qui soustendent l'accord sur les faits et les procédures considérées provisoirement comme vraies ou exactes.

Dans l'Antiquité grecque, Aristote distinguait déjà l'objet d'étude des «sciences théorétiques", le nécessaire, ce qui ne peut être autrement qu'il n'est, et celui des «sciences pratiques» qui, lui, est contingent et peut être autrement qu'il est. Même si 
nous avons appris entretemps que le nécessaire peut lui aussi être incertain ou comporter une validité provisoire ${ }^{5}$, la différenciation qu'opère Aristote est toujours probante.

Mais quelle formation donnons-nous alors à nos élèves pour approcher le contingent? Pour approcher tout ce qui peut être autrement qu'il est, pour décider quels projets réaliser et selon quels préceptes d'intervention? Pour décider des règles du vivre ensemble, d'y obéir (ou non) volontairement parce qu'elles sont (in)justifiées ? C'est pour répondre à cet objectif de formation qu'un cours d'éducation à la citoyenneté, voire un cours de «morale laïque » en France, prend tout son sens, n'en déplaise à N. de Condorcet.

En Belgique, par exemple, le cursus scolaire prévoit une éducation morale au sens large, qui peut être religieuse ou non confessionnelle, de 6 à 18 ans à raison de deux heures par semaine. L'éducation morale devient donc une discipline à part entière avec ses objectifs spécifiques. L'un des tout premiers objectifs d'un cours de morale non confessionnelle consiste d'ailleurs, selon nous, à mener les élèves à pouvoir distinguer la sphère de l'être (l'exact), celle du devoir-être (le bien, le juste) et celle du bien-être, c'est-à-dire la valeur ou le sentiment éprouvé à partir de l'être et du devoir-être (la valeur, le bon, le gout ${ }^{6}$ ). Cela revient à faire comprendre qu'il y a des faits sur lesquels on se met ou non d'accord, qu'il y a des normes sur lesquelles on se met ou non d'accord et qu'il y a des gouts et des valeurs qui ne se discutent pas mais qui sont censés exprimer ce que nous éprouvons chacun à propos de ce qui est et de ce qui doit être.

La spécificité éducative d'un cours d'éducation à la citoyenneté, à la moralité ou à la « morale laïque » en France, serait de développer chez nos élèves deux types de jugement qui ne sont pas systématiquement pris en charge par les autres disciplines, à savoir le jugement évaluatif et le jugement normatif. Le jugement évaluatif consiste à décider ce qui est souhaitable voire préférable, bref à évaluer, il porte sur le bien-être. Le jugement normatif, en revanche, consiste à décider ce qu'il faut faire pour bien faire ou pour être juste et se meut donc dans la sphère du devoir-être.

Le développement de ces deux types de jugement nécessite qu'on mette en place des dispositifs pédagogiques qui permettent d'atteindre cet objectif. Mais avant cela, il nous faut préciser quelle différence opérer entre les valeurs et les normes qui interviennent dans ces deux types de jugement: le jugement évaluatif évalue, pèse, les valeurs en présence ; le jugement normatif fait de même avec les normes disponibles.

\section{Faits, normes et valeurs}

Dans une perspective post-métaphysique, J. Habermas prend soin de montrer qu'un fait n'existe pas; il est le produit d'un accord (Habermas, 1987a), que ce soit celui d'une communauté des savants ou celui d'une cour de justice par exemple. Même si les faits interviennent à titre de raisons dans l'argumentation morale en général, nous allons surtout nous concentrer ici sur la distinction entre valeurs et normes et sur une typologie des normes.

\section{Les normes}

Tout d'abord, et surtout si l'article est lu par des philologues ou des grammairiens, le terme norme que j'utilise ici n'indique nullement ni une normalisation, ni une 
moralisation. Il est simplement synonyme d'énoncé normatif. Les énoncés normatifs (comme les énoncés prescriptifs d'ailleurs) se présentent sous la forme d'obligations ou d'impératifs, explicites ou implicites. Ils ne décrivent pas l'être mais indiquent un "devoir-être ». Ce devoir-être est déterminé en fonction d'un telos ${ }^{7}$ que l'on peut synthétiser sous la catégorie du «bien » ou du « juste». Car, comme le dit E. Kant (1943 : 59) : « Les seuls objets d'une raison pratique sont donc le Bien (Guten) et le Mal (Bösen) ».

21 Rappelons aussi que J. Habermas distingue la prétention à la vérité et la vérité. Ce qui revient à toujours avoir en tête qu'une norme peut prétendre à la justesse sans être juste, sans qu'elle soit validée ou acceptée comme telle. L'énoncé «X paie ses impôts » prétend dire quelque chose d'exact, mais, pour que cet énoncé soit vrai, il faudra en vérifier l'exactitude. De même, «il faut dire la vérité » est une norme sans qu'elle ne soit juste pour autant à priori. Quand il sera question de normes ou de normativité d'un énoncé, cela devra toujours s'entendre ici distinctement de moralisation.

Jürgen Habermas (1992 : chap. V) utilise le terme «norme » dans le sens fort d'obligation morale; il qualifie de «recommandation» l'impératif technique et de «conseil» l'impératif éthique.

Toutes les obligations, en effet, ne nous obligent pas de la même manière, avec la même force. Les normes de moralité peuvent être classées en trois catégories ${ }^{8}$ : les normes techniques, éthiques et morales ${ }^{9}$. La relativité de l'obligation est desservie par le sens relatif du verbe devoir dont la force illocutionnaire peut varier. Ainsi le devoir est-il faible pour les normes techniques parce qu'il n'y va, tout au plus, que d'efficacité («pour te rendre à tel endroit, tu dois prendre tel bus»); en revanche, la force d'obligation est catégorique pour les normes morales qui obligent tout citoyen du monde parce qu'il y va de l'humanité de l'homme (" Tu ne tueras point »). Enfin, le devoir est fort ou nul lorsqu'il s'agit de normes éthiques. En effet, il y va de la vie bonne, voire du salut, pour ceux que les normes éthiques concernent, tandis qu'elles sont indifférentes aux autres ( $\mathrm{Tu}$ adoreras Dieu seul et tu l'aimeras plus que tout »). Pour comprendre cette dernière difficulté, il faut souligner que la norme éthique est une règle de conduite qui a pour fonction d'atteindre un telos, une fin subjective, et que la norme n'oblige que ceux qui poursuivent cette fin-là. Les commandements religieux, par exemple, n'ont de force d'obligation que pour ceux qui veulent gagner leur "paradis » et n'engagent pas les autres qui ont une autre conception de l'existence ou de la «vie bonne ». Les questions éthiques, dit J. Habermas (1992: 99), n'exigent nullement une rupture complète avec la perspective égocentrique, «elles se rapportent en effet au telos d'une vie à chaque fois mienne ».

Notons qu'une même règle, par exemple «Je dois étudier", peut énoncer une norme technique (pour atteindre un but pratique: passer l'examen demain) et une norme éthique (pour me réaliser). Pareillement, une même règle, par exemple «Je dois porter assistance à personne en danger ", peut énoncer une norme éthique si elle fait obligation en fonction du telos que je veux atteindre (éviter par exemple le remords de mon indifférence à la détresse) ou une norme morale si elle fait obligation catégorique de préserver l'humanité de l'homme.

25 Le degré d'obligation des normes apparait aussi, lorsqu'elles sont coulées dans la forme du droit, notamment par le degré de sanction pénale qui est attachée à sa transgression. Le problème surgit quand il s'agit, pour vivre ensemble, de couler de telles normes techniques, éthiques et morales dans la forme du droit ${ }^{10}$. On peut supposer que les 
normes techniques seront aisément validées par les citoyens (et considérées à ce titre comme légitimes) si l'impératif d'efficacité est atteint et neutre sur le plan de la moralité. Rouler à gauche ou à droite est insignifiant sur ce plan pour autant que tout le monde se range à l'une ou l'autre option ${ }^{11}$.

Si les lois visent à préserver l'humanité de l'homme, il est raisonnable de penser qu'elles recevront l'aval ou le consentement de tous, en démocratie du moins. Tout au plus peut-il sans doute y avoir controverse, comme avec le principe de précaution, sur le fait qu'il y ait danger pour l'espèce.

Où le bât blesse, c'est en matière de normes éthiques. Car, si la loi civile élève au rang de l'humanité de l'homme une fin ou un telos particuliers, elle risque l'illégitimité, voire la partialité, rendant de surcroit injuste la sanction de l'infraction. Et si elle ne le fait pas, elle peut manquer pour certains à un devoir catégorique et rendre l'État laxiste. Ainsi s'expliquent les dures controverses sur les questions de l'avortement, de l'euthanasie, de l'homoparentalité, où l'éthos religieux diverge de l'éthos profane sur la conception naturelle, voire divine, ou culturelle de la vie.

La typologie des normes proposée ici ne vise pas seulement à la clarification sémantique pour que nous puissions nous comprendre entre chercheurs, elle a aussi une fonction politique. Si le législateur est tenu en démocratie de respecter le pluralisme éthique, il doit faire coexister pacifiquement des points de vue éthiques divergents et être capable, pour ce faire, de ne pas généraliser des convictions particulières. Cette typologie des normes a aussi une fonction déontologique pour les enseignants qui ont affaire, à des degrés divers, à l'éthique. Dans un État de droit, et donc à l'école publique, le pluralisme éthique doit être en principe respecté sauf si des obligations contradictoires se font jour. Dans ce cas, c'est la loi civile porteuse du consensus ou de la moralité publics qui l'emportera. Comme dans le cas du médecin qui juge nécessaire de procéder à la transfusion sanguine d'un mineur et qui, sans l'autorisation des parents témoins de Jéhovah, demandera un jugement en référé.

Le rôle déontologique de l'enseignant, lui, est :

1. de respecter les choix éthiques du jeune tout en lui faisant comprendre que le droit à la liberté de pensée est exigible par chacun à égalité et donc qu'il ne s'agit jamais de vouloir élever une obligation particulière en norme catégorique ;

2. de faire comprendre que la loi est parfois le garant de la coexistence pacifique de points de vue éthiques divergents ;

3. que la désobéissance à la loi, même pour des raisons éthiques, fait encourir à celui qui commet l'infraction la sanction prévue par la loi.

Cette fonction déontologique suppose bien évidemment que l'enseignant soit lui-même capable d'éviter l'empire de sa subjectivité et de ne pas généraliser ses propres normes éthiques sans toutefois sombrer dans le relativisme moral. C'est dire s'il est urgent de mettre en œuvre une formation, initiale ou continue, des enseignants dont l'État exigerait cette fonction.

\section{Les valeurs}

31 Le risque de moralisation est encore plus grand quand il s'agit de valeurs parce que cellesci sont indiscutables au sens où il n'est pas possible de les hiérarchiser de façon objective. Pourtant, beaucoup d'enseignants classifient les valeurs en bonnes ou mauvaises. 

situation de clarifier leurs valeurs et de les hiérarchiser sans que le groupe-classe ne discute à proprement parler ces choix. La fonction pédagogique de l'enseignant est d'amener l'élève à prendre conscience de ce qui l'anime et d'ainsi contribuer à ce qu'il se connaisse mieux, comme le recommande la maxime antique : «Connais-toi toi-même ». Sa fonction déontologique est de respecter les choix de l'élève et de les faire respecter par 
les autres dans le groupe-classe, ce qui revient à faire découvrir par tous qu'il n'y a pas à priori de bonnes ou de mauvaises valeurs. Le professionnel peut tout au plus faire découvrir par les leçons de l'expérience que certains choix sont plus judicieux que d'autres pour soi et pour le vivre ensemble. Cela revient à prendre la valeur comme le condensé d'une norme que l'expérience a validé ou non. À l'aune de l'expérience de l'humanité, la relativité des valeurs ne signifie nullement leur relativisme.

Encadré 1. Quelques exemples pédagogiques

- Pour clarifier les valeurs des élèves de 4 à 7 ans, l'enseignant peut recourir aux images : leur demander de choisir l'image qui a leur préférence et de pouvoir dire pourquoi. Pour leur faire hiérarchiser, leur demander de coller trois images (dont on a préalablement reconstruit, avec l'aide de l'enseignant, la valeur) selon leur ordre de préférence. Pour faire découvrir la relativité des valeurs et de leurs échelles, organiser une promenade devant l'exposition des tops 3 de la classe (Leleux, 2014 : leçon $n^{\circ} 5$ ). Cette activité a en outre l'avantage de faire ressortir pour toute la classe qu'une échelle de valeurs 1 (par exemple : famille, amour, santé), n'est pas meilleure qu'une échelle de valeurs 2 (santé, amour, famille) et qu'il n'est donc pas possible de les discuter voire de les coter.

- L'élève peut aussi être conduit à clarifier ses propres valeurs en opposition ou par comparaison avec celles d'un auteur. Pour ce faire, l'album peut être un support de même qu'un texte philosophique ou un article de journal. Par exemple, de manière à clarifier la morale dont les élèves sont les plus proches, nous avons conçu une leçon en plusieurs séquences (Leleux, 2010 : 179-198). L'objectif est, dans un premier temps, de faire retrouver par les élèves les valeurs défendues ou rejetées, explicitement ou implicitement, dans les différents textes (un extrait de l'Épitre de Paul aux Philippiens, des aphorismes de Ainsi parlait Zarathoustra, le chant de l' Internationale, une présentation de la morale laïque); dans un second temps, de faire élire trois valeurs par chaque élève qui doit les classer par ordre d'importance parmi toutes les valeurs retrouvées dans un des textes.

- Autre exercice, en éducation à la citoyenneté, pour différencier valeurs et normes, préférences personnelles et objectivité juridique : on peut demander aux élèves de lire la Déclaration universelle des droits de l'Homme et de sélectionner les cinq articles qui leur semblent les plus importants ou auxquels ils sont le plus attachés. Ils doivent ensuite dégager la valeur qui sous-tend ces cinq articles et les classer dans un top 5 de leurs valeurs. Cette activité fait alors apparaitre la différence entre le point de vue axiologique subjectif et l'ordre logique qui prévaut dans tout texte juridique (le point de vue objectif du Droit). Le participant, quel que soit son âge, découvre alors que sa préférence va à la liberté, à l'égalité, à la solidarité ou à la justice, à l'asile, à la liberté de culte, au procès équitable... que son attachement aux droits peut être d'ordre biographique et relié au « Connais-toi toi-même » dans un ordre qui n'est pas celui de la Déclaration qui servira de référence, lui, dans le cas d'un procès par exemple.

Le lecteur intéressé par ces protocoles pédagogiques est invité à se référer aux nombreuses leçons, séquences et activités que nous avons eu l'occasion de proposer ${ }^{13}$. 

faire découvrir les valeurs implicites ou explicites de tout texte, fût-ce d'un album de jeunesse, mettre le jeune en situation de décentration cognitive (différencier le point de vue de l'auteur et le sien propre) avant de l'amener éventuellement à comparer son échelle de valeurs à celle de l'auteur, sachant que l'une n'est pas meilleure à priori que l'autre.

\section{Développer le jugement normatif}

L'éducation morale au sens large est essentiellement une éducation au jugement normatif ${ }^{14}$, c'est-à-dire qu'il s'agit d'apprendre au jeune à décider d'obéir volontairement aux normes d'action parce qu'elles se justifient pour atteindre un but, une fin, voire préserver l'humanité de l'homme (prendre l'homme comme fin, dirait E. Kant).

Développer cette décentration cognitive et le jugement normatif des jeunes consiste en substance à :

1. leur faire réfléchir à ce qu'il convient de faire pour atteindre un but ;

2. leur apprendre à distinguer différents buts: un but simplement pratique (efficacité de l'action), un telos (une fin, c'est-à-dire le but d'une existence, le bonheur, la réussite d'une vie, le salut dans un au-delà de la vie terrestre...), une finalité humaine (humanité de l'homme) ;

3. leur faire hiérarchiser des normes d'action en fonction des différents objectifs, découvrir que certaines normes sont plus générales (incluant le point de vue subjectif) que d'autres (excluant d'autres points de vue subjectifs) ;

4. leur faire comprendre que le vivre ensemble dans un État de droit suppose de pouvoir généraliser une norme pour tous à égalité ;

5. leur faire reconstruire la nécessité d'une sanction attachée à la transgression d'une norme juridique ;

6. les amener à distinguer le légal et le légitime (une norme juridique n'est pas forcément juste) ;

7. leur faire découvrir qu'un désaccord avec le législateur peut prendre la forme d'une désobéissance civile, sachant que celle-ci peut entrainer une sanction.

Plusieurs études et recherches montrent que le développement du jugement normatif s'opère lorsque les élèves sont mis en situation de discuter avec leurs pairs de problèmes moraux. La théorie du développement du jugement moral de L. Kohlberg sert d'étalon de mesure de ce développement ${ }^{15}$. C'est la raison pour laquelle les dispositifs pédagogiques que nous proposons de mettre en œuvre pour développer un tel jugement recourent à l'échange d'arguments moraux entre les pairs.

Notons toutefois que les sujets de discussion, les normes discutées, peuvent diverger selon le type de cours : un cours de moralité publique, c'est-à-dire un cours d'éducation à la citoyenneté ou de morale laïque en France, ne prendra pas pour objet de réflexion les normes qui touchent à la morale personnelle, au salut ou aux convictions particulières. En revanche, celles-ci seront matière à discussion dans les cours de religion et de morale non confessionnelle. La discussion sera sans doute différente selon que l'on se réfère à une transcendance divine ou non. Dans un cours de morale non confessionnelle en tout cas, le parti pris sera de ne pas donner la priorité à un argument (d'autorité) d'ordre

Pratiques, 163-164 | 2014 
religieux. Par exemple, le devoir de charité ne ferait pas partie d'un tel cursus alors que le devoir de solidarité, sous la forme par exemple de la sécurité sociale, y serait questionné.

Plusieurs dispositifs pédagogiques peuvent être mis en place pour développer la décentration cognitive et l'échange des arguments moraux avec les pairs en ayant à l'esprit la typologie des normes que nous avons reconstruite plus haut.

Encadré 2. Quelques exemples de dispositifs pédagogiques

- Le premier dispositif pédagogique auxquel recourir est celui que L. Kohlberg a utilisé lui-même pour établir sa théorie du développement du jugement moral, à savoir le dilemme moral. On peut prendre l'expression dans son sens usuel mais, sur le plan pédagogique, le dilemme moral obéit à quelques caractéristiques qui le différencient d'une simple alternative (par exemple : « Faut-il faire ceci ou cela?»). Le dilemme moral est plus contraignant parce qu'il décrit une situation dans lequel le « héros » du récit, que l'on appellera « $X$ », se trouve face à deux issues contradictoires sans que l'une ne soit à priori meilleure que l'autre. L'exemple fameux cité par L. Kohlberg étant le dilemme de Heinz ${ }^{16}$ qui doit choisir entre voler le pharmacien pour guérir sa femme ou la laisser mourir faute d'avoir les moyens de pouvoir acheter le médicament. Sous cette réserve, bien des dilemmes peuvent constituer la base d'une discussion normative. Par exemple, le dilemme d'Aisha :

"Aisha est marocaine et sort avec Patrick. Une de ses amies lui apprend que Patrick fait partie d'une bande de "skins" et l'emmène dans un bistrot où ils se réunissent. Aisha assiste à une discussion dans un café entre Patrick et les "skins". Il y est question de venger un des leurs, grièvement blessé, en organisant un attentat dans un établissement où se réunissent les amis et frères de Aisha. Aisha tente d'en empêcher Patrick, sans succès. Plus, celui-ci la menace de représailles si elle diffusait le secret. L'attentat projeté est imminent.

Que doit faire Aisha, prévenir la police ou être fidèle à son ami ?»

Ce dilemme permet une réflexion approfondie sur le devoir de dénoncer ou non l'imminence d'un délit. Il n'y a pas de bonne issue à priori au dilemme dans la mesure où vont entrer en concurrence des arguments moraux différents, les uns relevant du devoir citoyen, les autres de l'amour ou d'une obligation conjugale. Les échanges devraient permettre de hiérarchiser ceux-ci, mais la discussion devrait aussi faire apparaitre que personne ne peut exiger de quelqu'un d'autre que luimême qu'il se sacrifie pour son prochain et endure des représailles. La « morale rationnelle scelle l'abolition du sacrifice », dit J. Habermas ${ }^{17}$.

L'intérêt de travailler avec des dilemmes en classe vise précisément non à transmettre une morale toute faite, mais à la réflexion et à l'échange de justifications pour développer le jugement normatif.

- C'est précisément pour développer un tel jugement qu'une discussion à visée philosophique (DVP) doit, selon nous, se donner pour objectif pédagogique que les élèves discutent de problèmes moraux, échangent des justifications au choix de telle ou telle norme d'action et produisent en conclusion (provisoire) de la discussion une sophia, une sagesse, un savoir pratique, éventuellement sous la forme d'un conseil à 
un ami. L'accent étant mis dans une telle pratique pédagogique sur le « que dois-je faire? ? (normatif) plutôt que sur le « qu'est-ce que ? » (descriptif).

Le lecteur intéressé pourra prendre connaissance d'une vingtaine de discussions à visée philosophique à partir de contes africains écrits par J. Lantier pour les élèves de 5 à 14 ans (avec leurs questions, les plans de discussion de l'enseignant et la production des sagesses des élèves) dans un ouvrage paru en 2010 (Leleux, Lantier, 2010).

- La régulation des émotions par la DVP est un programme de réflexion sur les émotions (Leleux, Rocourt \& Lantier, 2014) qui vise lui aussi à développer le jugement normatif. L'objectif général de ce programme est de développer l'autonomie affective des élèves et, ce faisant, de contribuer à la civilisation démocratique au sens où, comme le dit le sociologue N. Elias $(2002 ; 2003)$, celle-ci est le résultat d'un long processus de maitrise des affects. Processus durant lequel, selon lui, l'art militaire et le rapport de force physique a progressivement cédé la place à l'art de la parole. Le dispositif pédagogique a été totalement mis en place à l'école maternelle, en $2^{\mathrm{e}}$ année de primaire (2P, 7-8 ans), $6 \mathrm{P}$ (11-12 ans) et partiellement mis en œuvre en $1 \mathrm{P}$ (6-7 ans) et en $1^{\text {re }}$ année de secondaire $\mathrm{S}$ (1S, 12-13 ans). Il consiste, à partir d'Histoires d'émotions de Jan Lantier, d'identifier une émotion, d'en comprendre son utilité et ses inconvénients et de la réguler en conséquence par un jugement normatif. En maternelle, les élèves ont réfléchi à quatre émotions (la peur, la colère, la tristesse, la joie) ; en primaire et secondaire, ils ont identifié deux concepts (émotion et famille d'émotions) et réfléchi à quatre émotions de base supplémentaires (la surprise, le dégout, la honte et l'émoi amoureux), à une émotion mixte (la jalousie) et à une autre émotion sociale que la honte (la fierté).

- Les enseignants, qui n'ont pas en charge l'éducation morale ou citoyenne, mais qui ont à gérer le vivre ensemble d'une classe et à accomplir une des missions traditionnelles de l'école qui consiste à socialiser les jeunes, pourront développer le jugement normatif de leurs élèves par la réflexion à propos des droits et devoirs de chacun en classe, sachant qu'en démocratie, si tout droit est dû à chacun à égalité, chaque droit suppose un devoir afférent. Raison pour laquelle, cette réflexion en classe devrait être menée sous la forme d'un règlement ou d'une charte dans lesquels les droits sont confrontés aux devoirs correspondants et ne se limitent pas, comme c'est souvent le cas, à une liste d'interdits ou de devoirs (Leleux, 2006).

- À la suite de la réflexion précédente concernant les droits et les devoirs, l'enseignant pourra recourir à un dispositif inspiré de la pédagogie Freinet : le conseil de la classe qui est l'organe de législation et de décision de la classe sur le vivre ensemble (Leleux, 2008).

Quel que soit le dispositif utilisé, l'important pour l'enseignant, qu'il soit spécialisé en didactique de la morale et de la citoyenneté ou non, consiste à développer le jugement normatif de ses élèves. Qui dit « développement » dit aussi que ce jugement normatif doit tendre ou se confondre avec un jugement "postconventionnel », au sens de L. Kohlberg, c'est-à-dire un jugement normatif qui se fonde librement sur des principes pour justifier le choix d'une règle d'action. En l'absence de cette autonomie du jugement comme objectif pédagogique, l'action de l'enseignant serait une entreprise de moralisation visant 
à faire de ses élèves de bons exécutants, reniant du même coup l'idéal moral démocratique d'autonomie politique. Il est particulièrement nécessaire que les enseignants soient formés à éviter une telle dérive de moralisation, dérive qui donnerait raison à $\mathrm{N}$. de Condorcet selon lequel on ne pourrait confier l'éducation à l'école.

\section{BIBLIOGRAPHIE}

ARISTOTE (1992) : Éthique à Nicomaque, trad. du grec par J. Barthélemy Saint-Hilaire, revu par A. Gomez-Muller, Paris, Éd. Le Livre de poche.

BRUNER, J. (2010 [2002]) : Pourquoi nous racontons-nous des histoires? Le récit au fondement de la culture et de l'identité, Paris, Retz (coll. « petit forum »).

CONDORCET, N. (1791) : «Premier Mémoire. Nature et objet de l'instruction publique », in : Id., Cinq mémoires sur l'instruction publique, chapitre 5, p. 33 (en ligne : http://classiques.uqac.ca/ classiques/condorcet/cinq_memoires_instruction/Cinq_memoires_instr_pub.pdf, consulté le 10/03/15).

Elias, N. (2002 [1973]) : La civilisation des mœurs, trad. de l'allemand par P. Kamnitzer, Paris, Pocket (coll. « Agora »).

- (2003 [1975]) : La dynamique de l'Occident, trad. de l'allemand par P. Kamnitzer, Paris, Pocket (coll. « Agora »).

FERRY, J.-M. (1991) : Les puissances de l'expérience, Paris, Éd. du Cerf (coll. « Passages »), 2 vol.

HABERMAS, J. (1987a [1972]) « Théories relatives à la vérité », trad. par R. Rochlitz, in : ID., Logique des sciences sociales et autres essais, Paris, PUF, p. 282-283.

- (1987b [1981]) : Théorie de l'agir communicationnel, t. 1, Rationalité de l'agir et rationalisation de la société, trad. de l'allemand par J.-M. Ferry, Paris, Fayard.

- (1992 [1991]) : De l'éthique de la discussion, trad. de l'allemand par M. Hunyadi, Paris, Éd. du Cerf. HOFFMAN, M. (2008) : Empathie et développement moral. Les émotions morales et la justice, Grenoble, Presses universitaires de Grenoble.

KANT, E. (1943 [1788]) : Critique de la raison pratique, trad. par F. Picavet, Paris, PUF (coll. «Quadrige »).

LALANNE, J. (1990) : « Le développement moral cognitif chez Lawrence Kohlberg », Entre-vues, 7, p. 15-28.

LELEUX, C. (1997) : Repenser l'Éducation civique. Autonomie, coopération, participation, Paris, Éd. du Cerf (coll. « Humanités »).

- (2003) : « Théorie du développement moral chez Lawrence Kohlberg et ses critiques (Gilligna et Habermas) », in : J.-M. Ferry \& B. Libois (dirs), Pour une éducation postnationale, Bruxelles, 2003, Éd. De l'Université de Bruxelles (coll. « Philosophie et Société »), p. 11-128 (en ligne : http:// users.skynet.be/claudine.leleux/KohlbergPublDEA.pdf).

- (2006) : Éducation à la citoyenneté. Apprendre des droits et des devoirs de 5 à 14 ans, t. 2, Bruxelles, De Boeck (coll. « Outils pour enseigner).

- (2008) : Éducation à la citoyenneté. La coopération et la participation de 5 à 14 ans, t. 3, Bruxelles, De 
Boeck (coll. « Outils pour enseigner »).

- (2009) : «La discussion à visée philosophique pour développer le jugement moral et citoyen ? ", Revue française de pédagogie. Recherches en éducation, 166, janvier-février-mars, p. 71-87 (en ligne : http://rfp.revues.org/1271).

- (en collab. avec Rocourt, C.) (2010) : Pour une didactique de l'éthique et de la citoyenneté. Développer le sens moral et l'esprit critique des adolescents, Bruxelles, De Boeck. (coll. « Action »).

- (2014) : Hiérarchiser des valeurs et des normes de 5-14 ans, $3^{\mathrm{e}}$ éd., Bruxelles, De Boeck (coll.

«Apprentis citoyens »).

LeLEUX, C. \& LANTIER, J. (2010) : Discussions à visée philosophique à partir de contes pour les 5 à 14 ans, Bruxelles, De Boeck (coll. « Apprentis philosophes »).

LELEUX, C., ROCOURT, C. \& LANTIER, J. (2014) : Développer l'autonomie affective de 5 à 14 ans, Bruxelles, De Boeck (coll. « Apprentis citoyens »).

RAINVILLE, M. (1978) : Manuel pratique de formation à l'approche de Kohlberg, Québec, Université de Québec.

\section{NOTES}

1. "S'il est possible de déduire "U" du contenu normatif des présuppositions pragmatiques universelles de l'argumentation en général, l'éthique de discussion se laisse ramener à la formulation suivante, plus économe : (D) Chaque norme valide devrait pouvoir trouver l'assentiment de tous les concernés, pour peu que ceux-ci participent à une discussion pratique » (Habermas, $1992: 34$ ).

2. Principe « $U$ » d'universalisation et principe « $D$ » de discussion.

3. «Ainsi les concepts des trois mondes servent de système de coordonnées supposé en commun, et dans lequel les contextes situationnels peuvent être ordonnés de telle sorte qu'un accord soit obtenu sur ce que les participants peuvent traiter tantôt comme état de fait, tantôt comme norme valide, ou tantôt comme expérience vécue subjective » (Habermas, 1987b : 85-86).

4. "L'éducation publique doit se borner à l'instruction"; «Un autre motif oblige encore de borner l'éducation publique à la seule instruction ; c'est qu'on ne peut l'étendre plus loin sans blesser des droits que la puissance publique doit respecter» (Condorcet, 1791).

5. Contrairement à Aristote (1992 : livre IV, chap. 1, § 13) qui pensait qu'« il est impossible de délibérer sur un fait accompli ; on ne délibère que sur l'avenir et sur le possible, parce que ce qui a été, c'est-à-dire le passé, ne peut pas n'avoir point été ».

6. Nous laissons en suspens la question problématique du beau.

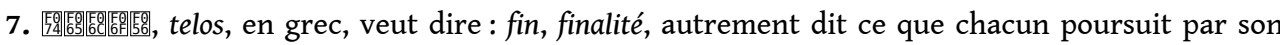
action : le bonheur, une existence réussie, le salut... et qui constitue le sens de sa vie.

8. La typologie des normes présentée ici peut être reconstruite à partir d'E. Kant et de J. Habermas.

9. J'utilise le terme de «norme » au sens de règle (du latin « norma » qui signifie « règle ») et je distingue ensuite les normes techniques, éthiques et morales dans le même sens que Kant différencie les impératifs hypothétiques (habileté et prudence) et catégorique [Emmanuel Kant, Critique de la faculté de juger [1790], trad. A. Philonenko, Paris, 1989, Vrin, p. 22].

10. Il faut alors distinguer, comme le font aussi E. Kant et J. Habermas, les normes de moralité et les normes juridiques, de légalité. Disons simplement ici que les normes juridiques peuvent elles aussi être déclinées dans les trois catégories (techniques, éthiques et morales).

11. En revanche, bousculer des habitudes en changeant le sens de la circulation serait d'un ordre de moralité supérieur. 
12. La qualité bonne ou mauvaise de quelque chose, la vertu ou le vice d'un caractère... ces attributs peuvent être positifs ou négatifs en fonction du plaisir ou du déplaisir escompté au regard de l'expérience (directe ou transmise).

13. Pour le secondaire, voir surtout C. Leleux (2010), et, pour le primaire, voir surtout C. Leleux (2014).

14. Plusieurs auteurs dont les défenseurs d'une morale de la sollicitude comme C.Gilligan refusent de réduire la maturité morale d'une personne à la maturité de son jugement moral. Et, de fait, l'expérience quotidienne, notamment en classe, nous amène continuellement à repérer aussi bien des attitudes de sollicitude, qui ne se fondent pas nécessairement sur des principes, que des raisonnements moraux qui ne sont pas suivis d'effet pratique. Toutefois, ce qui a été démontré par M. Rainville (1978:62), c'est « une bonne corrélation entre le développement du jugement moral » et l'action morale, comme, par exemple, « la résistance à l'obéissance, la résistance à la tentation de tricher, et la non-délinquance ». Dans le contexte scolaire, il est donc important, audelà de la controverse théorique que nous avons discutée ailleurs (Leleux, 2003), de viser au développement du jugement normatif. D'autant que déontologiquement, il n'entre pas dans la fonction de l'enseignant d'agir sur le comportement.

La maturité morale d'une personne dépend sans doute aussi largement de son empathie, voire de sa sensibilité envers son prochain. Mais, comme l'a démontré M. Hoffman (2008:276) lui-même, une personne empathique peut aussi être injuste en privilégiant par exemple quelqu'un de proche ou quelqu'un qui l'émeut au point que, selon lui, « la moralité empathique seule, risque de ne pas suffire ». Il est possible toutefois, du point de vue cognitif, de faire prendre à l'élève le point de vue de l'autre, de le faire se décentrer, sans verser dans une forme de chantage ou de culpabilisation qui serait d'ailleurs contre-productive sachant qu'il existe, comme le décrit M. Hoffman lui-même, le phénomène de "surexcitation empathique ", c'est-à-dire un état dans lequel l'individu se défend et se détourne de l'empathie, parce qu'elle est trop douloureuse pour lui.

15. Par exemple : J. Lalanne (1990) a montré qu'une argumentation, pour justifier le choix d'une issue au dilemme moral, peut évoluer d'un stade $n$ vers une argumentation de stade $n+1$; C. Leleux (2009).

16. Pour ce dilemme et l'utilisation qu'en fait L Kohlberg, voir, par exemple, C. Leleux (1997 : $55-69 ; 2008$.

17. «La validité des commandements moraux est liée à la condition que ceux-ci soient, d'une manière générale, suivis comme étant le fondement d'une praxis universelle. [...] Ce n'est qu'alors que les commandements moraux sont dans l'intérêt commun et qu'ils ne posent pas - précisément parce qu'ils sont également bons pour tous - d'exigences surérogatoires. Dans cette mesure, la morale rationnelle scelle l'abolition du sacrifice » (Habermas, 1992 : 125).

\section{RÉSUMÉS}

Dans la mesure où nous sommes tenus de présupposer que tout agir communicationnel prétend à l'entente, l'éthique est à la base de tout savoir. Or, peu d'enseignants sont formés à l'enseignement de l'éthique et de la citoyenneté. La présente contribution, après avoir clarifié quelques concepts fréquemment utilisés dans la discipline d'éducation à la moralité, ciblera deux objectifs de la didactique spécifique (développer les jugements normatif et évaluatif) et de la 
déontologie spécifique (respecter le pluralisme éthique et éduquer sans moraliser, c'est-à-dire éduquer à l'autonomie du jugement).

So far as we are hold to presuppose that each communicative action pretends to an agreement, ethics are at the base of every knowledge. But not many teachers are trained to give an education in ethics and citizenship. This article first clarifies some concepts frequently used in the education of morals. Afterwards it aims at two targets of the specific didactic (to develop the prescriptive judgment and the judgment of values) and the specific professional ethics (to respect the ethical pluralism and to educate without moralizing, that means to educate to an autonomous judgment).

\section{INDEX}

Mots-clés : éthique, citoyenneté, jugement normatif, jugement évaluatif, normes et valeurs

Keywords : ethics, citizenship, prescriptive judgment, judgment of values, norms and values

\section{AUTEUR}

\section{CLAUDINE LELEUX}

Catégorie pédagogique Defré, Haute École de Bruxelles 